\title{
Enhancing the properties of some inverse opal metamaterials by the stopband control
}

\author{
Daniela Ionescu $^{1, *}$ and Irina Macovei $^{2}$ \\ 1 "Gh. Asachi" Technical University of Iasi, Department of Telecommunications and Informational \\ Technologies, Carol I Blvd. 11, 700506 Iasi, Romania \\ 2 "Gr. T. Popa" University of Medicine and Pharmacy of Iași, Faculty of Pharmacy, Universitatii Str., \\ No. 16, 700115 Iasi, Romania
}

\begin{abstract}
The inverse opal structures present very good mechanical and optical properties, like high mechanical strength and Young modulus, important photoluminescence and stimulated Brillouin scattering. Enhancement of the optical properties of the metamaterials based on different types of inverse opal can be obtained by controlling the stopband of the $3 \mathrm{D}$ periodic structure. $\mathrm{SiO}_{2}, \mathrm{TiO}_{2}$ and $\mathrm{CeO}_{2}$ photonic crystals with inverse opal structures have been studied, presenting voids of $100-280$ $\mathrm{nm}$. The stopband dependence on the physical and geometrical parameters of the matrix has been analyzed (ions nature, voids diameter, photoelastic constant). The study was performed by structural simulation methods, using the HFSS program. The test configuration was set for the visible light domain. The effective refraction index $n$ and the stimulated Brillouin scattering coefficient $g_{P}$ have been determined and represented on parametrical graphs. Results were compared with the theoretic calculated values. By varying different structural parameters and the stopband control, superior values of the optical parameters have been obtained, in comparison with the data given in literature. We report an increasing of the photoluminescence with about $7 \%$, respectively and enhancement of the stimulated Brillouin scattering coefficient of $11 \%$ when parameters are correlated.
\end{abstract}

\section{Inverse opal metamaterials}

The inverse opal structures were demonstrated to present very good mechanical and optical properties, like high mechanical strength and Young modulus, important photoluminescence and stimulated Brillouin scattering. Due to their property of periodic modulation of the refraction index onside the material, the photonic crystals based on inverse opals are used for multiple applications. In optics and optoelectronics, devices based on stimulated Brillouin scattering with silicon, like Brillouin lasers and compact microwave signal processors have been realized. The (bio) sensors design and biological applications were another challenge using this type of materials. The inverse opal metamaterials are also used for the energy storage and communications, in solar cell

${ }^{*}$ Corresponding author: danaity@yahoo.com 
technology, for electrochemical energy storage, at super capacitors, fuel cells, catalysts [12]. The applications are based of programmable properties of these 3D structured materials, which can be controlled by varying the physical and geometrical parameters of the samples and the applied fields.

\subsection{Characteristics - improving the properties by the stopband control}

Enhancement of the optical properties of the metamaterials based on different types of inverse opal can be obtained by controlling the stopband of the $3 \mathrm{D}$ periodic structure. The stopband or pseudo gap represents an incomplete photonic band gap, characteristic of this type of materials, where the light propagates in only some directions. These properties of prohibiting the electromagnetic wave propagation depend on the refraction index contrast and the lattice topology. The purpose is to manipulate the light propagation in a controllable way. The control parameters are internal and also external, depending on the applied fields and incident wave.

A detailed analysis of the wave propagation phenomena through the metamaterials was performed, considering the structure at lattice level. The heterostructure with voids and the unit cell configuration of the inverse opals were illustrated in Figure 1. The lattice constant of inverse opals can be of thousands of nanometers, while the sphere diameters and void, of hundreds of nanometers (e.g.: for $\mathrm{SiO}_{2}$ inverse opal, lattice constant is about $2300 \mathrm{~nm}$, having spheres of 400-600 $\mathrm{nm}$ and void of about $200 \mathrm{~nm}$ ).

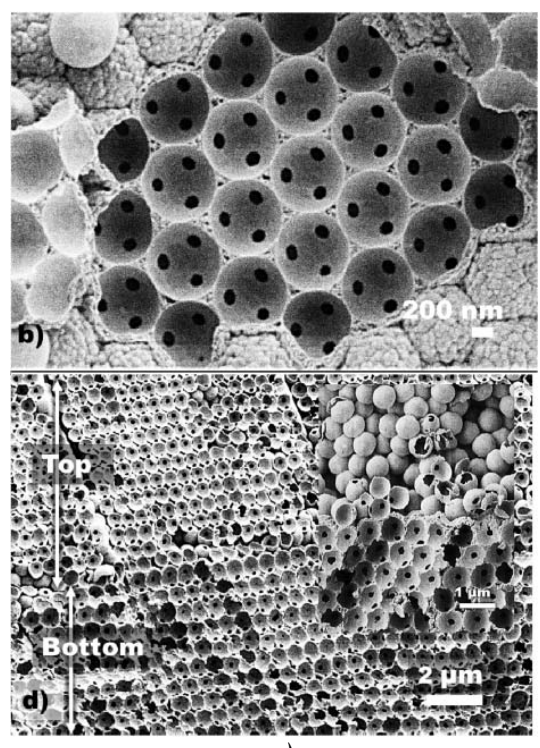

a)

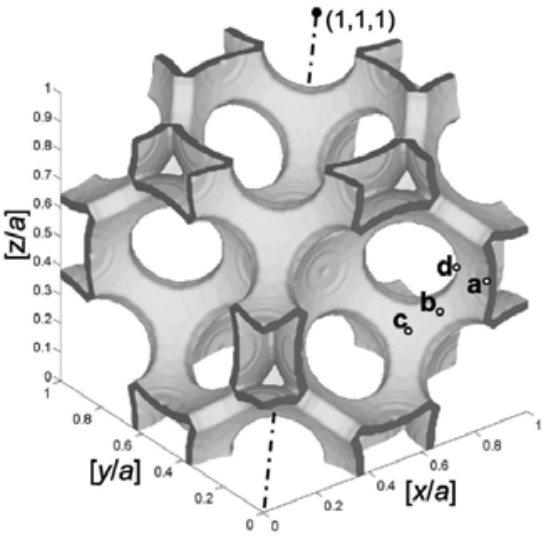

b)

Fig. 1. a) Inverse opal $\left(\mathrm{TiO}_{2}\right)$ heterostructure with voids (after Lee \&Optical Materials Express 3(8), 2013); b) Unit cell of the inverse opal photonic crystal (after I. Nikolaev \&, JOSA B 26(5), 2009).

The structural analysis points out dependences of the materials stopband on: ions nature, voids diameter, photoelastic constant, effective refraction index, external parameters: angle of the propagating light. Literature reports that the stopband width is controlled by the refraction index contrast, while the stopband position can be shifted by modifying the average refraction index ( $\lambda_{\text {stopband }}$ is increasing when the average refractive index decreases), the lattice parameter or the diameter of opal spheres [2-3]. Also the stopband can be shifted ( $\lambda_{\text {stopband }}$ is increasing) when the angle of light illumination increases (e.g. the blue shift of $\{111\}$ stopgaps of $\mathrm{SiO}_{2}$ inverse opals with increasing angle of incidence) [4-5]. 


\subsection{Theoretical considerations}

For the inverse opals, the stopband wavelength can be calculated using the modified Bragg formula, as follows $[2,6]$ :

$$
\lambda_{\text {stopband }}=2 \cdot 0.816 \cdot D \cdot \sqrt{n_{\text {eff }}^{2}-\sin ^{2} \theta}
$$

Where $D$ is the opal spheres diameter, $n_{\text {eff }}$ is the effective refraction index and $\theta$ is the angle between the direction of incident light and the normal to the (111) plane of $f c c$ crystal lattice. The effective refraction index of the inverse opal structures can be calculated as:

$$
n_{\text {eff }}{ }^{2}=(1-f) \cdot n_{\text {air }}{ }^{2}+f \cdot n_{\text {medium }}{ }^{2}
$$

Where $n_{\text {air }}$ and $n_{\text {medium }}$ represent the refraction indices of the air and solid medium of the opal respectively, $f$ is the filling fraction of the opal. For example, a value for the $\mathrm{SiO}_{2}$ inverse opal can be $n_{\text {eff }} \approx 1.13$.

Wave propagation phenomena in inverse opals are governed by the stimulated Brillouin scattering (SBS), which is characterized by a power gain coefficient given by:

$$
g_{P}=\frac{2 \pi n^{7} p_{12}^{2}}{c \lambda^{2} \rho_{0} v_{a} \Delta v_{B}}
$$

Where $p_{12}$ is the longitudinal elasto-optic coefficient, $\rho_{0}$ is the material density, $\lambda$ is the wavelength (of the testing resonant field), $v_{a}$ is the longitudinal acoustic wave velocity within the material ( $v_{a}=\sqrt{c_{33} / \rho_{0}}, c_{33}$ is the elasticity modulus), $\Delta v_{\mathrm{B}}$ is the line width of spontaneous Brillouin scattering (full width at half maximum, FWHM), $n$ is the refractive index and $c$ is the vacuum velocity of light [6]. For example, a group of values to calculate the Brillouin gain $g_{\mathrm{P}}$ for $\mathrm{SiO}_{2}$ inverse opal are: refraction index of silica $n=1.444$; the elasto-optic constant of $\mathrm{SiO}_{2}: p_{12}=0.285$; density $\rho_{0}=2.21 \cdot 10^{3} \mathrm{~kg} / \mathrm{m}^{3}$; wavelength $\lambda=$ $1.55 \mu \mathrm{m}$; acoustic wave velocity $v_{\mathrm{a}}=5996 \mathrm{~m} / \mathrm{s} ;$ FWHM $\Delta v_{\mathrm{B}}=28 \mathrm{MHz}$; the Brillouin frequency shift $v_{\mathrm{B}}=2 n v_{\mathrm{a}} / \lambda=11.1 \mathrm{GHz}$. Results a $g_{\mathrm{P}}$ of $4.52 \times 10^{-11} \mathrm{~m} / \mathrm{W}$ for this type of silica $\left(\mathrm{SiO}_{2}\right)$ inverse opal [6].

The material steady-state photoluminescence $P L$ can be estimated with the Elliott formula [7-8]:

$$
P L(\omega)=\operatorname{Im}\left\{\sum_{\lambda} \frac{F_{\lambda} S_{\lambda}}{E_{\lambda}-\hbar \omega-i \gamma_{\lambda}(\omega)}\right\}
$$

Where $\omega=2 \pi f$ is the angular frequency; $F_{\lambda}$ is the oscillator strength of the excite state $\lambda ; E_{\lambda}$ is the Eigen energy of the exciton state; $\hbar \omega$ is the exciting photon energy; $\gamma_{\lambda}$ is the dephasing constant associated with the exciton state $\lambda$, and $S_{\lambda}$ represents the spontaneousemission source contribution:

$$
S_{\lambda}=\sum_{\mathbf{k}}\left|\phi_{\lambda}(\mathbf{k})\right|^{2} f_{\mathbf{k}}^{e} f_{\mathbf{k}}^{h}+\Delta N_{\lambda}
$$

With $\mathbf{k}$ represents the wave vector, $\varphi_{\lambda}(\mathbf{k})$ is the exciton Eigen function, $f_{\mathbf{k}}{ }^{e} f_{\mathbf{k}}{ }^{h}$ term defines the probability to find an electron and a hole with same wave vector $\mathbf{k}$ (a correlated pair), $\Delta N_{\lambda}$ is the number of truly bound electron-hole correlated pairs in the material [7-8]. 
Literature reports that the bulk SBS gain coefficient of silicon can be enhanced by two orders of magnitude using a metamaterial design based on silicon inverse opals, due to larger compressibility of the inverse opal, which lowers the acoustic velocity and the Brillouin line width is reduced $[2,7]$. Our simulation methods have the purpose to correlate these internal and external parameters in order to obtain a stopband control and consequently an improving of the electro-optical properties of the metamaterial samples.

\section{Results for the optical parameters of the metamaterial}

$\mathrm{SiO}_{2}, \mathrm{TiO}_{2}$ and $\mathrm{CeO}_{2}$ photonic crystals with inverse opal structures have been studied here, presenting voids of $100-280 \mathrm{~nm}$ (lower at $\mathrm{SiO}_{2}$, higher values for $\mathrm{TiO}_{2}$ ). The considered ionic radii were: for $\mathrm{Si}: 0.054 \mathrm{~nm}$, for Ti: $0.0745 \mathrm{~nm}$, respectively for Ce: $0.101 \mathrm{~nm}$.

In visible range, the stop band positions were reported: for $\mathrm{SiO}_{2}$ inverse opals, $\lambda_{\text {stopband }}=$ $546 \mathrm{~nm}$; for $\mathrm{TiO}_{2}$ inverse opals, $\lambda_{\text {stopband }}=430 \mathrm{~nm}$, respectively $480 \mathrm{~nm}$; the $\mathrm{CeO}_{2}$ inverse opals presents photonic band gap at $485 \mathrm{~nm}$. These band gaps have been reproduced and studied by specifically methods.

The study was performed by structural simulation methods, using the HFSS program. The test configuration was set for the visible light domain. The simulation set-up, based on a multi-mode channel waveguide, having dimensions of $W=2.8 \mu \mathrm{m}, H=1.6 \mu \mathrm{m}$, is illustrated in Figure 2. The considered wavelength domain for analysis was: $470-680 \mathrm{~nm}$ (in visible spectrum).

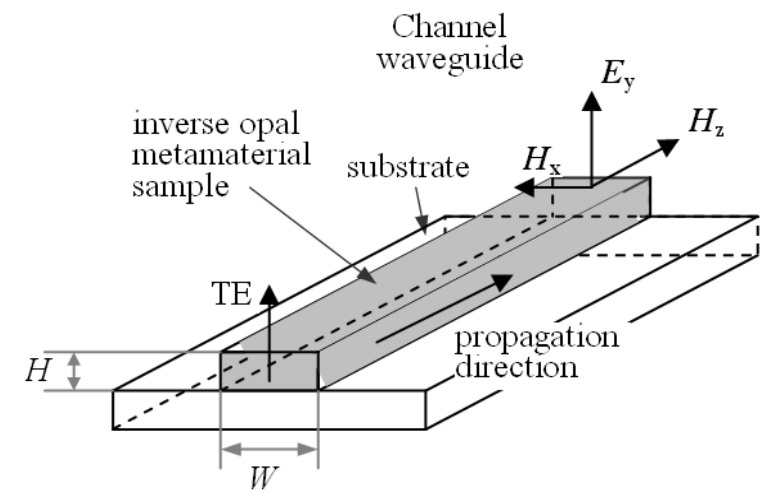

Fig. 2. Waveguide with inverse opal metamaterial sample.

Different parameters have been varied in order to modify the SBS gain and the metamaterials stopband. The effective refraction index $n$ and the stimulated Brillouin scattering coefficient $g_{P}$ have been determined and represented on parametrical graphs. Results were compared with the theoretic calculated values in case of the considered metamaterial structures.

Graphs for the stimulated Brillouin scattering coefficient $g_{\mathrm{P}}$ in function of voids diameter, $D_{v}$, are indicated in Figure 3. One observes an increasing of the gain for bigger voids materials, due to an enhanced scattering process inside the structure. In comparison with the results given by theory (indicated by the dotted curves), maxima were evidenced on graphs, at $D_{v}$ values of about multiple of the main element ionic radii ( $\mathrm{Si}$, Ti, respectively $\mathrm{Ce}$ ), which can be exploited for the stopband control. Graphs for the SBS coefficient $g_{\mathrm{P}}$ in function of stopband wavelength shifting, $\Delta \lambda_{\text {stopband }}$, are indicated in Figure 4. When the stopband position is shifted by internal parameters control ( $D_{v}$ and $n_{\text {eff }}$ have been modified and chosen for maximal effect), the gain can be increased, and making the most used $\mathrm{SiO}_{2}$ inverse opal very attractive for electro-optic applications. 


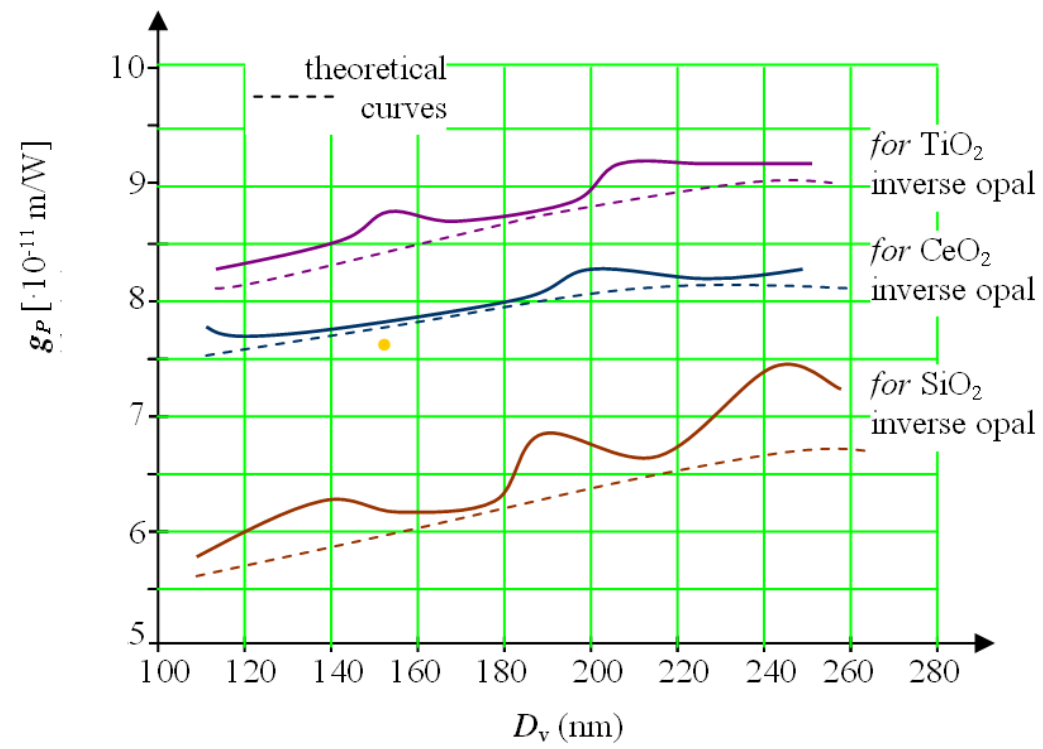

Fig. 3. Evolution of the stimulated Brillouin scattering coefficient, $g_{\mathrm{P}}$, in function of the voids diameter $D_{\mathrm{v}}$ of the inverse opal metamaterials. Simulation maxima are evidenced on graphs.

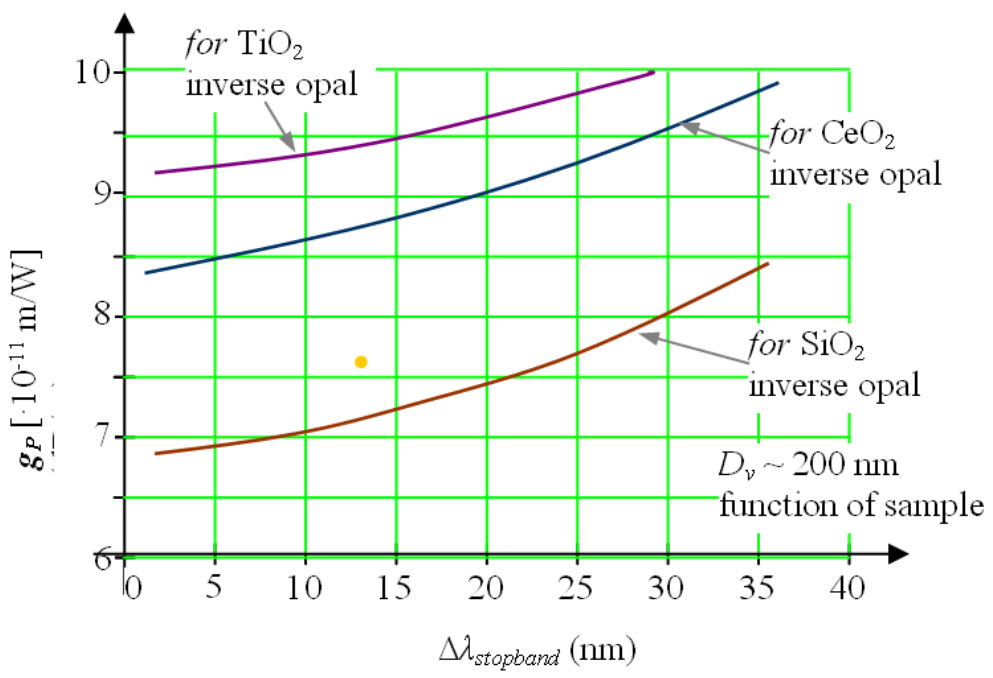

Fig. 4. Evolution of the stimulated Brillouin scattering coefficient, $g$, in function of the stopband wavelength shifting, $\Delta \lambda_{\text {stopband }}$ for the considered inverse opal metamaterials.

Analyzing the physical properties of considered inverse opal metamaterials, we have been illustrated in Figure 5 the graphs for the photoluminescence intensity in function of stopband wavelength shifting, $\Delta \lambda_{\text {stopband }}$. A photoluminescence increasing can be obtained for a moderate increasing of the stopband shifting, conclusion which is very convenient in practice. If the stopband shifts consistently, the photoluminescence is also enhanced in comparison with the basic values, but energy is consumed for material tuning. Materials with high refraction index (like $\mathrm{Ti}, n=2.153$ ) and high refraction index contrast present high photoluminescence, which increases fast when $\lambda_{\text {stopband }}$ is increased, and keep itself in the maximal range for a large interval of $\Delta \lambda_{\text {stopband }}$ values, but the maximum value is reached for higher stopband shifting than in the case of the other considered metamaterials. 


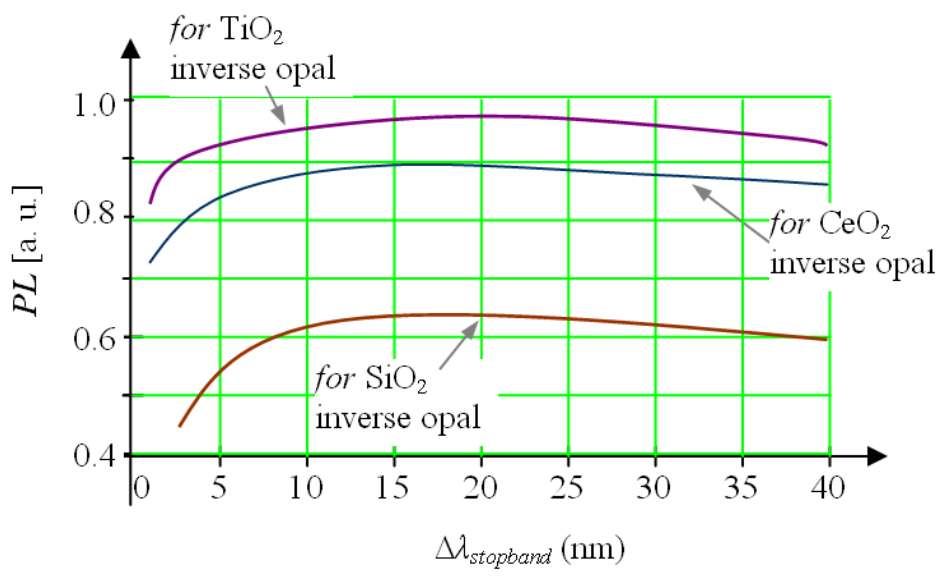

Fig. 5. Evolution of photoluminescence, $P L$ (in arbitrary units), in function of the stopband wavelength shifting, $\Delta \lambda_{\text {stopband }}$ for the considered inverse opal metamaterials.

\section{Conclusions}

In the case of some inverse opal metamaterials, the stopband dependence on the physical and geometrical parameters of the matrix has been analyzed (ions nature, voids diameter, photoelastic constant, refraction index, etc.). A tight dependence between the structural parameters and physical properties has been evidenced, intermediated by the stopband tuning. By varying different structural parameters and the stopband control, superior values of the optical parameters have been obtained, in comparison with the data given in literature. We report an increasing of the photoluminescence with about $7 \%$, respectively and enhancement of the stimulated Brillouin scattering coefficient of $11 \%$ when parameters are correlated.

\section{References}

1. Y. Hao, R. Mittra, FDTD Modeling of Metamaterials, Theory and Applications, (Artech House, Boston/London, 2009)

2. E. Armstrong, C. O'Dwyer, Artificial opal photonic crystals and inverse opal structures - fundam. and appl. from optics to energy storage, J. Mater. Chem. C 3, 6109 (2015)

3. C.-Y. Kuo, S.-Y. Lu, S. Chen, M. Bernards, S. Jiang, Stop band shift based chemical sensing with three-dimensional opal and inverse opal structures, Sensors and Actuators B: Chemical 124(2), pp. 452-458 (2007)

4. M. Curti, C. B. Mendive, M. A. Grela, D. W. Bahnemann, Stopband tuning of $\mathrm{TiO}_{2}$ inverse opals for slow photon absorption, Materials Research Bulletin 91 (2017)

5. G.J. Harrison, J.M. Arnold, Nigel Johnson, Angular dependence of the stopband of an inverse opal structure, Conference on Lasers and Electro-Optics Europe, Oct. 2000, Conference Digest (2000)

6. B. Stiller, Brillouin scattering in photonic crystal fiber: from fundamentals to fiber optic sensors, thesis, Université de Franche-Comté, France (2011)

7. R. J. Almassy, A. B. Kaye, T. M. Fletcher, Optical Modulation Utilizing Structures Including Metamaterials, Patent No. US 8,655,189 B2 (2014)

8. R. J. Elliott, Intensity of Optical Absorption by Excitons, Physical Review 108 (6), pp. 1384-1389 (1957) 